\title{
Treatment Strategy after Incomplete Endoscopic Resection of Early Gastric Cancer
}

\author{
Sang Gyun Kim \\ Department of Internal Medicine and Liver Research Institute, Seoul National University College of Medicine, Seoul, Korea
}

Endoscopic resection of early gastric cancer is defined as incomplete when tumor cells are found at the resection margin upon histopathological examination. However, a tumor-positive resection margin does not always indicate residual tumor; it can also be caused by tissue contraction during fixation, by the cautery effect during endoscopic resection, or by incorrect histopathological mapping. Cases of highly suspicious residual tumor require additional endoscopic or surgical resection. For inoperable patients, argon plasma coagulation can be used as an alternative endoscopic treatment. Immediately after the incomplete resection or residual tumor has been confirmed by the pathologist, clinicians should also decide upon any additional treatment to be carried out during the followup period. Clin Endosc 2016;49:332-335

Key Words: Therapeutics; Incomplete resection; Endoscopic submucosal dissection; Early gastric cancer

\section{INTRODUCTION}

Endoscopic resection has now been accepted in South Korea as the definitive treatment for cases of early gastric cancer (EGC) that indicate it, mainly because endoscopic techniques and accessories have improved, and because the widespread national cancer screening program has increased detection of EGC. Furthermore, the number of EGC cases that indicate endoscopic submucosal dissection (ESD) and for which this procedure is considered has risen, because many technical difficulties have been overcome, and because ESD has become the treatment of choice for EGC that indicate it. Moreover, it has been necessary to expand the use of ESD, because the general population is aging, and quality of life after treatment for EGC is an important consideration.

Received: May 2, 2016 Revised: May 23, 2016

Accepted: May 24, 2016

Correspondence: Sang Gyun Kim

Department of Internal Medicine and Liver Research Institute, Seoul National University College of Medicine, 101 Daehak-ro, Jongno-gu, Seoul 03080, Korea Tel: +82-2-740-8112, Fax: +82-2-743-6701, E-mail: harley1333@hanmail.net

(cc) This is an Open Access article distributed under the terms of the Creative Commons Attribution Non-Commercial License (http://creativecommons.org/ licenses/by-nc/3.0) which permits unrestricted non-commercial use, distribution, and reproduction in any medium, provided the original work is properly cited.
However, the increased use of ESD to treat EGC should not be allowed to jeopardize patient survival. Therefore, ESD should only be used to treat EGC in cases where (1) the risk of lymph node or distant metastasis is negligible, (2) complete resection is feasible, (3) the risk of complications is minimal, and (4) the chances of survival are comparable to those of surgical resection. Nonetheless, as more cases of EGC are considered as indicating ESD, the rate of incomplete resection has increased with the expansion of indication of ESD for EGC. Of course, incomplete resection does not necessarily indicate residual tumor; nonetheless, additional treatment should be considered in patients with who are at high risk of residual tumor, whereas patients at low risk of residual tumor can simply be followed up closely.

\section{COMPLETE RESECTION}

The term "complete resection" means that no tumor cells remain after treatment-this can be revealed by both endoscopy and histopathology. However, as microscopic synchronous tumor cannot be found without total mapping of the stomach, tumor-negative lateral and vertical resection margins are usually interpreted as indicating complete resection. Micro- 
scopic synchronous tumor is reported to exist in $7.4 \%$ of cases despite complete resection. ${ }^{1}$

In cases of piecemeal resection, completeness cannot be confirmed by ambiguous reconstruction of the specimen. In addition, in cases of irregular tumor arrangement or insufficient resection margin (less than $2 \mathrm{~mm}$ ), residual tumor may not be detected. False-negative tumor margins can also occur when the specimen is mapped in an incorrect direction or with a wide interval.

The specimen should be flattened and fixed immediately after resection, because the tissue may otherwise contract, leading to a false-positive result. Similarly, false-positive tumor margins can be caused by the cautery effect, whereby microscopic tumor cells around the resection margin are ablated.

The depth of tumor invasion, as well as the distance from the vertical resection margin, can be measured using pathologic mapping. When the tumor invades more than $500 \mu \mathrm{m}$ into the submucosal layer, the risk of lymph node or distant metastasis increases, irrespective of whether resection is complete. ${ }^{2}$ Furthermore, lymphovascular tumor invasion confers an increased risk of lymph node or distant metastasis. Put another way, the risk of residual tumor, or of lymph node/distant metastasis, increases with deeper submucosal or lymphovascular tumor invasion, irrespective of whether resection is complete.

Conversely, it may be that no residual tumor is found during follow-up without additional treatment in cases of incomplete resection, because false-positive tumor margins often occur. In addition, it is possible that no lymph node/distant metastasis has occurred in cases of deeper submucosal or lymphovascular tumor invasion. A resection is defined as curative when there is (1) no residual tumor, (2) no lymph node/distant metastasis, and (3) no additional treatment during long-term follow-up; this definition is conferred irrespective of whether resection is complete, and without regard for either depth of submucosal invasion or presence of lymphovascular invasion.

\section{RISK FACTORS FOR RESIDUAL TUMOR}

Tumor-positive resection margin is a risk factor for residual tumor. In two retrospective studies, residual tumor was found in $29 \%$ of patients with tumor-positive lateral resection margin. Additional risk factors for residual tumor were: piecemeal resection, diffuse type histology, and tumor-positive verticalor vertical and lateral-resection margin., ${ }^{3,4}$ However, residual tumor was found in only $5.8 \%$ of patients with mucosal cancer and tumor-positive lateral-only resection margin, implying that curative resection had been achieved in most such patients. ${ }^{5}$ Conversely, residual tumor was found in $20 \%$ of patients with submucosal tumor invasion, even though resection was complete; therefore, submucosal tumor invasion is a strong risk factor for residual tumor, irrespective of whether resection is complete. ${ }^{6}$

It has been reported that the risk factors for residual tumor are: large tumor size, extent of tumor invasion at the lateral resection margin, and undifferentiated histology. ${ }^{7-11}$ Therefore, in patients who display these signs, as well as those that show tumor-positive resection margin, additional treatment should be considered.

\section{RISK FACTORS FOR LYMPH NODE METASTASIS}

It is well known that tumor size, depth of tumor invasion, and undifferentiated histology are risk factors for lymph node
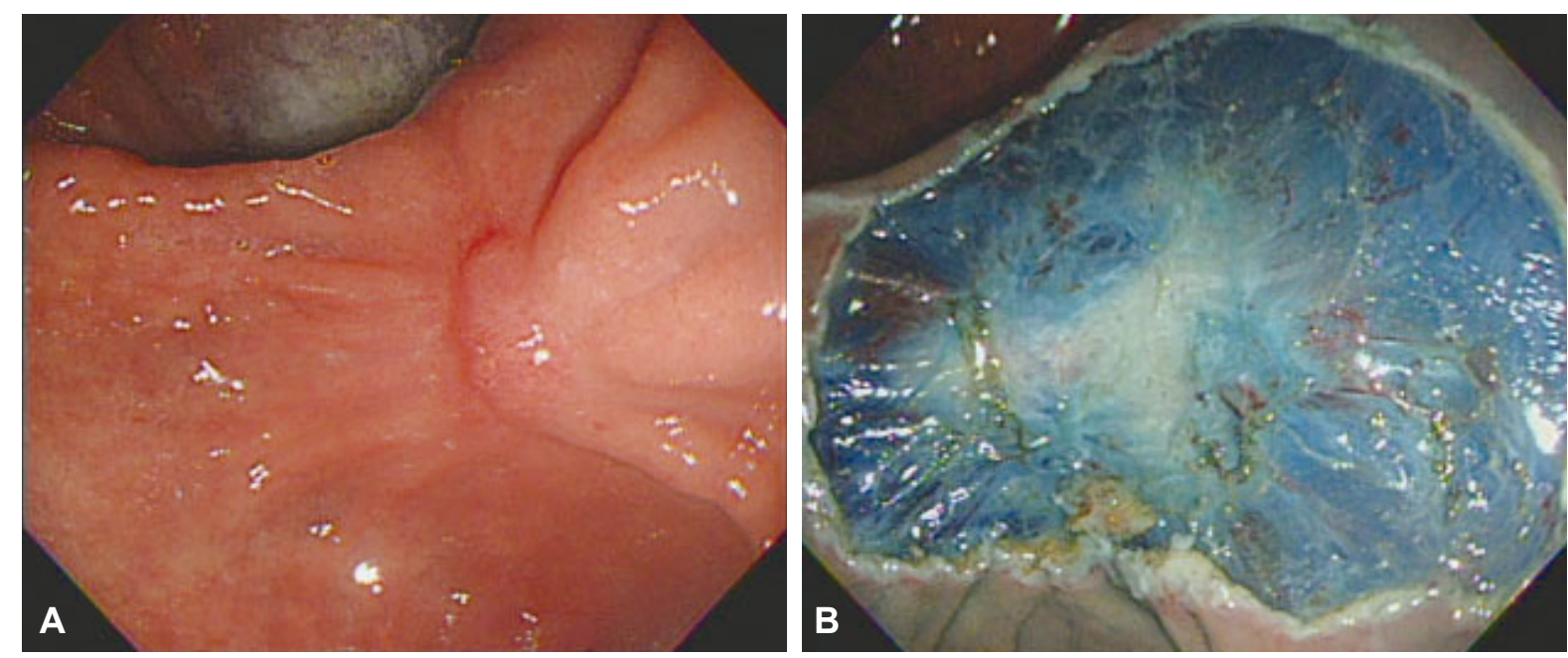

Fig. 1. Additional endoscopic submucosal dissection (ESD) to treat residual tumor during follow-up. (A) Residual tumor during follow-up after incomplete ESD. (B) Additional ESD for residual tumor, which may be technically difficult due to extensive submucosal fibrosis. 

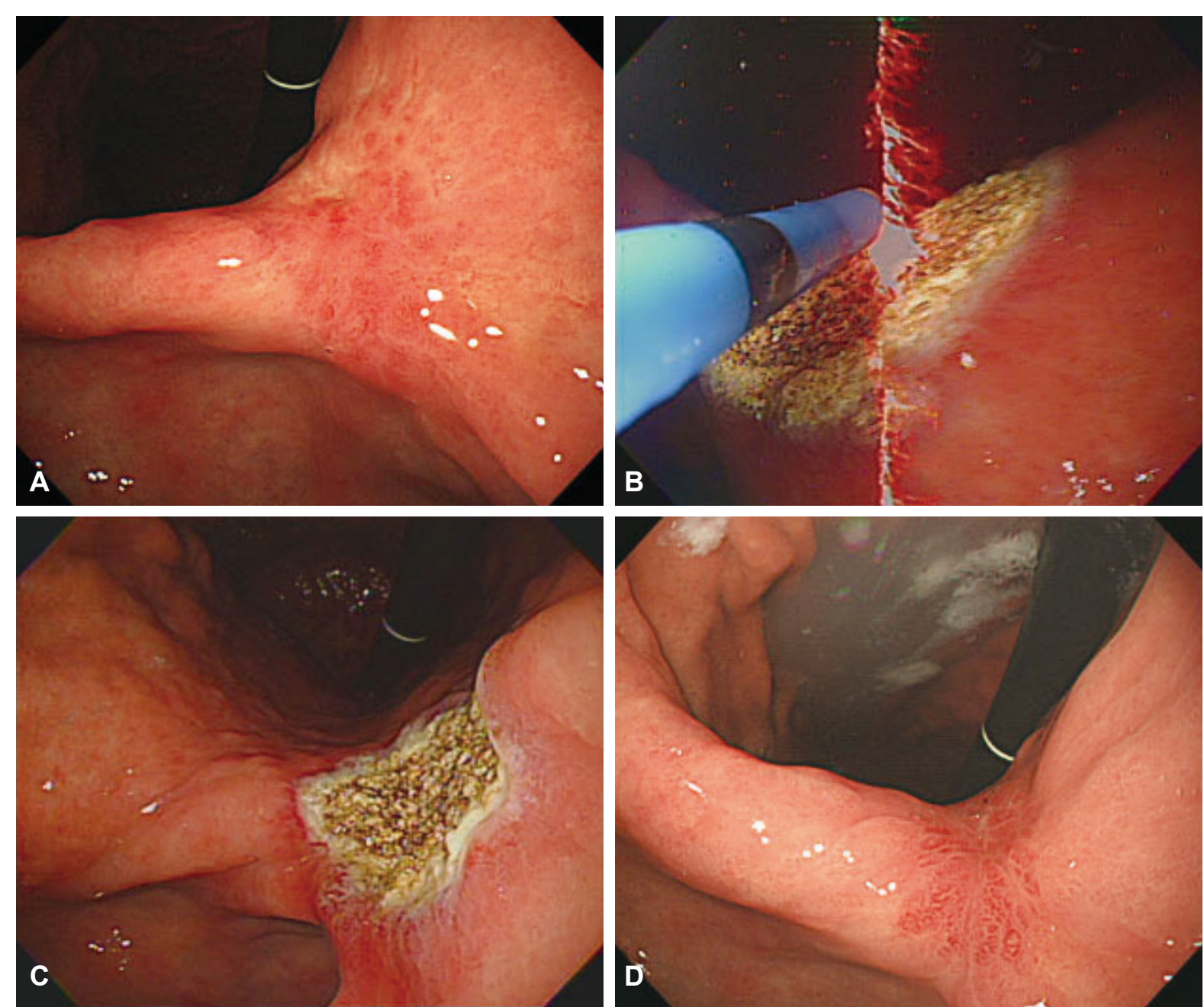

Fig. 2. Additional argon plasma coagulation (APC) to treat residual tumor during follow-up. (A) Residual tumor during follow-up after incomplete endoscopic submucosal dissection. (B) Additional APC for residual tumor. (C) Post-coagulation state. (D) No residual tumor during follow-up.

metastasis. ${ }^{2,12-15}$ For this reason, curative resection cannot be confirmed in cases with a high risk of lymph node metastasis, even if resection is complete. To illustrate, in one retrospective study, the rate of complete resection was significantly lower, and rates of submucosal and lymphatic tumor invasion were significantly higher, in patients with undifferentiated histology than in patients with differentiated histology, and residual tumor was found in $40 \%$, and lymph node metastasis in $13 \%$, of patients who had undergone additional surgical resection. ${ }^{16}$ According to the expanded criteria for ESD, when there is a high risk of lymph node metastasis, additional surgical resection is needed, irrespective of whether resection is complete. ${ }^{3,7,17,18}$

\section{TREATMENT STRATEGY AFTER INCOMPLETE RESECTION OF EGC}

Clinicians should decide on treatment strategy after incom- plete resection of EGC based on the tumor differentiation, tumor size, depth of tumor invasion, tumor involvement at resection margin, and lymphovascular tumor invasion. When mucosal cancer is less than $2 \mathrm{~cm}$ and there is microscopic tumor involvement at the lateral resection margin, but no definite gross residual tumor or lymphovascular tumor invasion, the patient should be closely followed up without immediate additional treatment, because the cautery effect may have caused a false-positive result. However, in cases of extensive tumor involvement at the lateral resection margin, large tumor size, or undifferentiated histology, additional treatment should be considered, because of the high risk of residual tumor.

Immediate additional ESD should be used when tumor involvement at the lateral resection margin has been confirmed using histopathological mapping; however, the disadvantages of such an approach are that the location of the residual tumor may be ambiguous, and that there may be no residual tumor 
(false-positive result). Additional surgical resection should be considered in cases with a high risk of lymph node metastasis, irrespective of whether resection is complete; that is, when there is large tumor size, submucosal or lymphovascular tumor invasion, or undifferentiated histology.

Additional ESD can be performed to manage any residual tumor found during follow-up; however, this may be technically difficult because of submucosal fibrosis (Fig. 1). When curative resection is expected to be difficult using additional ESD, surgical resection must be used. Alternatively, argon plasma coagulation (APC) can be used to ablate the tumor when ESD or surgical resection is contraindicated because of old age or severe comorbidity, or when the case involves residual adenoma rather than cancer (Fig. 2). However, as APC may not totally ablate the residual tumor, serial follow-up should be performed, and repetitive APCs may be required.

As tumor-positive vertical resection margin indicates extensive submucosal tumor invasion and a high risk of lymph node metastasis, additional surgical resection is mandatory in such cases. Moreover, additional surgical resection should be considered in cases that go beyond the expanded criteria, irrespective whether resection is complete because such cases involve a high risk of lymph node or distant metastasis. Specifically, in a retrospective study, the tumor had recurred during follow-up in $10.1 \%$ of such patients after ESD beyond the expanded criteria. ${ }^{19}$

\section{CONCLUSIONS}

The criteria for ESD to treat EGC have been expanded as endoscopic technical progress has been made; for this reason, the rate of incomplete resection has also increased. When planning treatment strategy, clinicians should consider the quality of life and long-term survival of patients, as well as the risk of residual tumor and lymph node metastasis.

\section{Conflicts of Interest}

The author has no financial conflicts of interest.

\section{REFERENCES}

1. Kitamura K, Yamaguchi T, Okamoto K, et al. Clinicopathologic features of synchronous multifocal early gastric cancers. Anticancer Res 1997;17(1B):643-646.
2. Gotoda T, Yanagisawa A, Sasako M, et al. Incidence of lymph node metastasis from early gastric cancer: estimation with a large number of cases at two large centers. Gastric Cancer 2000;3:219-225.

3. Oda I, Gotoda T, Sasako M, et al. Treatment strategy after non-curative endoscopic resection of early gastric cancer. Br J Surg 2008;95:14951500.

4. Lee JH, Kim JH, Kim DH, et al. Is surgical treatment necessary after non-curative endoscopic resection for early gastric cancer? J Gastric Cancer 2010;10:182-187.

5. Nagano H, Ohyama S, Fukunaga T, et al. Indications for gastrectomy after incomplete EMR for early gastric cancer. Gastric Cancer 2005;8:149154.

6. Song KY, Hyung WJ, Kim HH, et al. Is gastrectomy mandatory for all residual or recurrent gastric cancer following endoscopic resection? A large-scale Korean multi-center study. J Surg Oncol 2008;98:6-10.

7. Kim TK, Kim GH, Park do Y, et al. Risk factors for local recurrence in patients with positive lateral resection margins after endoscopic submucosal dissection for early gastric cancer. Surg Endosc 2015;29:2891-2898.

8. Hwang JJ, Park KJ, Park YS, et al. A scoring system for patients with a tumor-positive lateral resection margin after endoscopic resection of early gastric cancer. Surg Endosc 2016;30:2751-2758.

9. Lee JH, Lee JH, Kim KM, Kang KJ, Min BH, Kim JJ. Clinicopathological factors of multiple lateral margin involvement after endoscopic submucosal dissection for early gastric cancer. Surg Endosc 2015;29:3460-3468.

10. Sekiguchi M, Suzuki H, Oda I, et al. Risk of recurrent gastric cancer after endoscopic resection with a positive lateral margin. Endoscopy 2014;46:273-278.

11. Yoon H, Kim SG, Choi J, et al. Risk factors of residual or recurrent tumor in patients with a tumor-positive resection margin after endoscopic resection of early gastric cancer. Surg Endosc 2013;27:1561-1568.

12. Soetikno R, Kaltenbach T, Yeh R, Gotoda T. Endoscopic mucosal resection for early cancers of the upper gastrointestinal tract. J Clin Oncol 2005;23:4490-4498.

13. Miyata M, Yokoyama Y, Okoyama N, et al. What are the appropriate indications for endoscopic mucosal resection for early gastric cancer? Analysis of 256 endoscopically resected lesions. Endoscopy 2000;32:773778.

14. Abe N, Sugiyama M, Masaki T, et al. Predictive factors for lymph node metastasis of differentiated submucosally invasive gastric cancer. Gastrointest Endosc 2004;60:242-245.

15. Yokota T, Ishiyama S, Saito T, et al. Lymph node metastasis as a significant prognostic factor in gastric cancer: a multiple logistic regression analysis. Scand J Gastroenterol 2004;39:380-384.

16. Kang HY, Kim SG, Kim JS, Jung HC, Song IS. Clinical outcomes of endoscopic submucosal dissection for undifferentiated early gastric cancer. Surg Endosc 2010;24:509-516.

17. Ryu KW, Choi IJ, Doh YW, et al. Surgical indication for non-curative endoscopic resection in early gastric cancer. Ann Surg Oncol 2007;14:34283434.

18. Noh H, Park JJ, Yun JW, et al. Clinicopathologic characteristics of patients who underwent curative additional gastrectomy after endoscopic submucosal dissection for early gastric cancer or adenoma. Korean J Gastroenterol 2012;59:289-295.

19. Choi JY, Jeon SW, Cho KB, et al. Non-curative endoscopic resection does not always lead to grave outcomes in submucosal invasive early gastric cancer. Surg Endosc 2015;29:1842-1849. 